\section{'Striped TR signal': an intriguing Doppler echocardiography artefact or a pathological correlate?}

\section{CASE SUMMARY}

A 51-year-old man presented with complaints of insidious onset, gradually progressive dyspnoea on exertion NYHA (New York Heart Association) class II associated with paroxysmal palpitations. There was no history suggestive of rheumatic activity, rash, joint pains, haemoptysis, chest pain, cough, embolic phenomenon, intravenous drug abuse, cyanosis or similar illness in family. Clinical examination revealed a loud S1, opening snap and soft mid-diastolic murmur at the apex suggestive of mitral stenosis. ECG showed normal sinus rhythm, right axis deviation, left atrial enlargement and right ventricular enlargement. Echocardiography showed presence of thickened mitral valve leaflets with anterior mitral leaflet doming and posterior mitral leaflet restriction (mitral valve area $=1.3 \mathrm{~cm}^{2}$ ), normal left ventricular size and contractility, mild mitral regurgitation, mild tricuspid regurgitation (TR) and a normal aortic valve. There was no evidence of infective endocarditis. On colour Doppler interrogation of the TR jet, the spectral signal had the striking appearance of 'tiger stripes' on both sides of the baseline, constituting bright tissue signatures arising probably due to abnormal septal tricuspid leaflet oscillatory motion with the jet of $\mathrm{TR}^{1}$ (figure 1). These band-like signals appear to be associated with intracardiac oscillating structures, with the first band (lowest frequency on the Doppler recording) representing its fundamental frequency. These structures vibrate with a single frequency with several harmonic overtones. Differential diagnosis of tiger stripes includes valve regurgitation, flail prosthetic valve leaflet and possibly Lambl's excrescences as previously reported. ${ }^{2}$

\section{Ankit Kumar Sahu, Aditya Kapoor}

Department of Cardiology, Sanjay Gandhi Postgraduate Institute of Medical Sciences, Lucknow, Uttar Pradesh, India

Correspondence to Dr Ankit Kumar Sahu, Department of Cardiology, Sanjay Gandhi Postgraduate Institute of Medical Sciences, Lucknow 226014, Uttar Pradesh, India; ankitsahu.md@gmail.com

Contributors AKS: acquired the data and prepared the draft. AK: played an important role in planning the case report, concept, design and interpretation of the data.

Competing interests None.

\section{Patient consent Obtained.}

Provenance and peer review Not commissioned; externally peer reviewed.

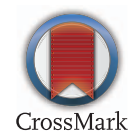

To cite Sahu AK, Kapoor A. Heart Asia 2016;8:45. doi:10.1136/heartasia-2016010841

Heart Asia 2016;8:45. doi:10.1136/heartasia-2016-010841

\section{REFERENCES}

1 Kerut EK. Tiger stripes. Echocardiography 2007;24:558-9.

2 Davogustto G, Fernando RR, Loghin C. Lambl's excrescences, migranous headaches and "Tiger stripes". Tex Heart Inst J 2015;42:70-2.
Figure 1 Continuous-wave spectral Doppler image recording is shown. Note the bright tissue signatures resembling 'tiger stripes' (arrows) within the tricuspid regurgitation jet.

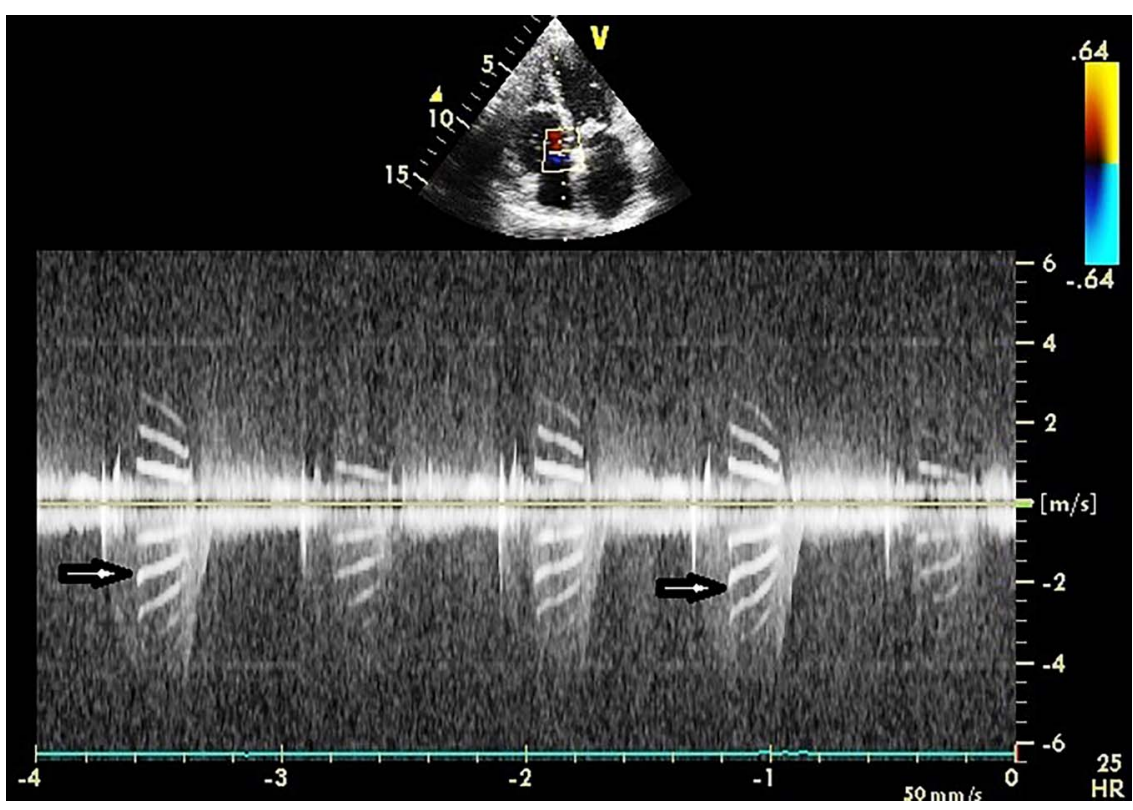

